\section{An Adodress}

\section{THE PLACE OF SURGERY IN THE TREATMENT OF PEPTIC ULCER.* \\ BY}

D. P. D. WILKIE, M.Ch., F.R.C.S.,

PROFESSOR OF SURGERY IN THE CNIVERSITY OF EDINBURGH.

THE choice of this subject for my lecture requires a little explanation, if not apology. The question of the advantages of medical over surgical treatment of peptic ulceration, and vice versa, has been debated almost ad nauseam. My object in this paper is not so much to uphold one line of treatment against another as to review the facts regarding the etiology of chronic ulceration so far as we know them, and to formulate rational lines along which the physician and surgeon, working hand in hand, may determine in which class of case, and when, medical measures should give place to surgery.

It is no new discovery in medicine that peptic ulceration will frequently yield to rest, frequent fluid feeds, and alkalis; nor, on the other hand, is it altogether surprising that extensive gastric resections are survived and followed by comparative comfort. What the patient with peptic ulceration wants to know is, what type of treatment will in his case restore him to health in a reasonable time with a probable chance of his remaining well under something approaching ordinary dietary and working conditions.

\section{Etiologi of Ulceration.}

Few subjects have engaged the attention of so many experimental research workers as this. It has been shown that gastric ulceration may be produced by many factors, singly or combined. Blood infection may produce multiple acute ulcers, deranged or interrupted nerve supply may lead to ulceration, retrograde venous embolism may occasionally cause ulcer. Buchner has recently shown that by a combination of starvation and the injection of histamine into rats gastric ulceration occurs regularly. As in the human subject, it occurs not in the acid-secreting part of the organ, but in the part immediately adjacent to this. Rowlands, Underhill, and.. Mendel, and recently Magee, Anderson, and McCallum, have shown that ulceration may appear in the stomach as one of the manifestations of deficiency disease, and Rogoff and Stewart that it may follow adrenalectomy, an operation which, as Dale has shown, produces a condition of the blood not unlike that following injection of large doses of histamine.

It has always been difficult in the experimental animal to maintain ulceration and to get the typical deep punchedout chronic ulcer found in the human subject. Further, in this particular case it is difficult to produce experimentally conditions which are quite comparable to those in the human being. We are therefore thrown back to a large extent upon cur clinical and pathological observations on the human subject. We reflect on the fact that the condition is met with in relatively healthy young adult males; that its symptoms are, to begin with, strictly periodic; that hyperacidity is present in the early stages; that painful spasm, as evidenced by the relief obtained by swallowing anything, is the rule. Further, we remark that dental infection is a frequent but by no means constant accompaniment, and that in many cases there is a history of acute or chronic appendicular trouble. We find that constipation, worry, overwork, loss of sleep, excessive smoking, and irregular meals, particularly if these are of the carried or snack-counter type, all seem to precipitate or aggrarate the condition, which is more common among the highly strung and nervous than among the more phlegmatic members of the community. We note that, whilst multiple acute ulcers may be widespread, the chronic variety is found in certain strictly localized sites along the lesser currature of the stomach, especially at the point where the body of the stomach abuts on the pyloric canal, and in the first part of the duodenum, approximately half * Delivere! to the Hyde Division of the British Medical Association
on January 24th, 1929; illustrated by lantern slides. an inch beyond the pylorus, and, in the latter case, usually multiple, one on the anterior and one on the posterior wall. We have good grounds for stating that the cause of gastric and duodenal ulcers must be one and the same. as they are so frequently found together. In my own series of duodenal ulcers a coincident gastric ulcer was present in 15 per cent., and of gastric ulcers a coincident duodenal ulcer was presënt in over 50 per cent. of cases. That a certain degree of gastritis accompanies and probably precedes uloeration is a fact ' of 'experience. Postmortem room observations show that multiple scars of healed ulcers are not uncommon, and frequently alongside an active penetrating ulcer are found the scars of other more superficial and healed ulcers.

What is the initial and primary factor in determining the ulcer? I believe that it is a blood-borne--infection which settles in the lymph follicles of the stomach and duodenum, some of which soften and erupt on the surface, the devitalized tissue undergoing digestion and leaving small follicular ulcers. The infection is streptococcal in type, and the primary source is usually the teeth or tonsils, and possibly, in some cases; the appendix. My reasons for this belief are as follows: In many cases we have recovered a streptococcus in pure culture from the base of an excised ulcer. In a number of cases we have found a coincident streptococcal infection of the gall-bladder and the appendix, and, whilst we have not been able to demonstrate experimentally the same selective affinity of the streptococcus for the stomach and duodenum that we have for the gall-bladder, we have sufficient evidence to point to the probability of this being the case.

There is admittedly a great tendency for acute ulcers in the stomach and duodenum to heal. Why is it that a certain number of such ulcers develop into the chronic peptic variety, and why are they found in such definite restricted areas of the stomach and duodenum? If we examine the common ulcer sites we find two characteristic features-first, the mucosa is relatively fixed, and secondly, the blood supply is relatively precarious. The greater curvature of the stomach, which contains few lymph follicles and the mucosa of which is lax and mobile in order to accommodate itself to the changing capacity of the organ, is practically immune from ulceration. The lesser curvature, on the other hand, is rich in follicles, is comparatively a fixed part, and is capable of little expansion; a defect in its lining remains open and is, moreover, in the direct traffic route of the entering food. The first part of the duodenum is, like the stomach, part of the foregut, has a smooth, unfolded mucous membrane rich in lymph follicles, and receives the acid chyme practically undiluted.

If these facts explain to some extent the site of chronic ulceration, what determines chronicity? Clinically we recognize two features in ulcer cases-hyperacidity and spasm. Further, we know that factors such as worry, excessive smoking, etc., which upset the nervous system, may, and frequently do, contribute to the onset of an "attack" of ulcer symptoms. From radiological studies we learn that in the duodenal ulcer cases rapid initial emptying of the stomach occurs, and during this period the patient is free from discomfort. A simall residue is left in the stomach; this is hyperacid, pyloric spasm sets in, and the patient experiences the typical gnawing pain which he can relieve to some extent by swallowing fluid or food, which will cause pyloric relaxation. The majority of duodenal ulcers are small and shallow, and if given rest and shielded from the hyperacid gastric chyme, the tondency to healing is well marked. In the case of gastric ulcer acid appears to play a less important part, indeed hypo-acidity is present in many cases of chronic ulcer; hypermotility is also less pronounced and pyloric spasm comes on earlier after taking food. The drag on the irritated lesser curvature after taking food induces painful local spasm, which handicaps the blood supply and does not favour healing. The pain is notably relieved by the recumbent attitude, which obviates or lessens the drag.

It is a notable feature in the history of ulcer cases that a country holiday will almost always dispel an attackthat is to say, fresh air, sunlight, and a change of diet have an inmediate beneficial influence. In reviewing the etiology of peptic ulcer we must therefore consider, 
I believe, three main factors: infection, nervous influences, and a possible dietary vitamin deficiency. In this connexion Mr. Garnett Wright has suggested that the success of the modern medical treatment of ulcer depends on the correction of vitamin deficiency by the giving of raw eggs, cream, etc., to patients who were accustomed to live on tea, white bread, cakes, and tinned or cooked foods.

In the light of the facts stated above we can understand how the removal of septic foci in the mouth, rest in the recumbent position, the ensuring of adequate sleep, the cutting down or out of tobacco, the giving of small meals of fresh vitamin-rich fluid food at frequent intervals, and the supply of alkali, may together favour, indeed almost ensure, the healing of gastro-duodenal ulceration in all but old-standing and neglected cases.

The Rationale of Surgical Treatment.

Apart from emergency operations for perforated ulcer, operative treatment for peptic ulcer is a practice of not many years' duration. It may be said to have started when it was found what great relief followed a gastroenterostomy for irremovable cancer of the pylorus. The earlier operations were all done to relieve a mechanical difficulty-that is, a stenosis resulting from the cicatrization of an ulcer in the duodenum or stomach. The brilliant results of such operations induced surgeons to try the same short-circuiting operation in cases of ulcer without stenosis in order to give rest to the ulcerated part. It was further found that the short-circuit permitted the entry of a certain amount of bile, mixed with pancreatic and duodenal secretion, into the stomach, and thus reduced acidity. This, the so-called physiological action of the stoma, was held by many, notably Paterson, to be the most beneficent effect of the operation. The results of gastro-enterostomy for chronic duodenal ulcer with some degree of mechanical difficulty from stenosis - that is, with a somewhat dilated stomach-were almost uniformly good. In a few regurgitant vomiting from a badly placed stoma resulted, and in a small percentage (less than 3 per cent.) a peptic ulcer developed at the stoma.

In another group, however, in which an irritable ulcer without stenosis but with marked hyperacidity and pronounced gastric hypermotility was present, the results of the short-circuiting operation were by no means so favourable. Here the danger of jejunal ulcer is a very real one, and only the most careful and prolonged after-treatment will prevent it. The consequence has been that an injudicious selection of cases for the operation, resulting in the development of jejunal ulceration in an unpleasantly large group of individuals, has prejudiced the good name of one of the most beneficent of surgical procedures.

The case of gastric ulcer is altogether different. After a few years of trial gastro-enterostomy was found to be most uncertain in its results in the treatment of gastric ulcer. Almost 50 per cent. of patients continued to have symptoms, and in a few cases malignant disease supervened on the simple ulcer which had not healed. As with duodenal ulcer, so with the smaller non-penetrating gastric ulcers, healing will result in a large proportion of cases under systematic medical treatment. When the ulcer is large and penetrating, however, even prolonged medical treatment will fail in many instances to cure, and during this long and tedious course of treatment malignant disease may have supervened and spread to the regional lymph glands or beyond them. The rule of the Mayo Clinic, that all penetrating gastric ulcers of $1 \mathrm{~cm}$. or more in diameter must be regarded as potentially malignant and should be removed, is one that I would havo our ultra-conservative and optimistic medical friends take to heart.

Size, penetration, and tendency to stenosis and hourglass constriction should, in my opinion, be the indicatiors for surgical intervention.

\section{The Bleeding Ulcer.}

After a patient has had one or more severe haemorrhages the question of surgical intervention usually arises. The problem presents itself first as to the risk run by persevering with purely medical measures, and, secondly, as to the immediate risk of operation and the possibility of dealing with the bleeding ulcer in such a way as to give a reasonable hope of lasting cure. To maintain that a patient never dies of haemorrhage from an ulcer is to go contrary to facts. Any pathologist of experience can cite very many cases where haemorrhage from an ulcer was the immediate cause of death. The operation for haemorrhage during the acute and threatening stage is, however, not only a dangerous, but in my experience an unsatisfactory, one. More often than not the haemorrhage has stopped at the time of operation, no definite bleeding point can be dealt with, and the patient, even if transfused, is not in a fit condition to allow of the radical treatment which would appear to be the only rational means of ensuring against recurrence. I believe it is wiser to tide the patient over the immediate effects of the haemorrhage, taking the risk of another, possibly fatal, bleeding, and to operate not earlier than ten days after the last severe bleeding. If no radiographic examination has been made prior to the bleeding it is usually wiser to wait until this can be done, for if it shows no gross deformity, either in stomach or duodenum, it is questionable if any operation should be performed.

For the bleeding duodenal ulcer a short-circuiting operation may be successful, but in my experience it fails in about 40 per cent. of cases to prevent recurrence of bleeding sooner or later. The persistently bleeding duodenal ulcer is usually on the posterior wall, is penetrating, and shows little tendency to heal after a short-circuiting operation. It is in this class of case that a partial duodenectomy is, to my mind, indicated, and I would reserve this operation for this type of ulcer. If the patient's general condition or the technical difficulties make such radical treatment undesirable a temporary closure of the pylorus and a gastro-enterostomy may be carried out.

For the bleeding gastric ulcer local excision with a short-circuit or a partial gastrectomy is required. In both cases the suitable operation is no minor one, and should not be attempted until the patient's condition is such as to make the immediate risk little if at all greater than that of any major gastric operation.

\section{Conservative Medicine versus Radical Surgery.}

In this country at the present time there is unquestion. ably a strong trend in favour of prolonged medical treatment of peptic ulcers, no matter at what stage or of what size the ulcers may be. The writings of MacLean and Hurst have done much to stimulate a renewed interest and zeal in the purely medical lines of treatment. This swing of the pendulum was perhaps inevitable after a period in which operative treatment was resorted to without mucli nicety of discrimination and selection of cases, with its inevitable aftermath of unsatisfactory results. On the Continent, on the other hand, the tendency has been in the direction of ever more radical surgery. It has gone so far that in some clinics a total extirpation of the "ulcer-bearing area" is the standard treatment for duodenal as well as for gastric ulcer. Some surgeons, with a naive disregard for the facts of physiology, remove what they term the acid-secreting part of the stomach with the object of avoiding a jejunal ulcer. As Buchner has so clearly pointed out, ulcers are found not in the acidsecreting parts, but in those which, whilst not secreting acid, are exposed to acid content.

\section{The Argument.}

If we examine the basis of the argument of the ultraconservative school against operative treatment we find that it rests on their experience with the surgical derelicts -the few with regurgitant vomiting, and the rather larger number with jejunal ulcer. Also we find that they have a very natural aversion to the sacrifice of a large part of a most useful and highly specialized organ because of the presence on its wall of a small infective ulcer. Further, they state that, given time and care, even the largest gastric ulcers will heal under medical treatment.

On the other side their more surgically minded brethren point to the large number of the populace who suffer from chronic ulcer and who cannot or will not carry out the prolonged medical treatment and still more prolonged dietetic regime which some physicians advocate so strongly. 
Further, they emphasize the real danger of overlooking early malignant disease if chronic penetrating gastric ulcers are to be subjected for an indefinite period to persistent and unrelenting medical measures.

\section{The Via Media.}

In this, as in so many fields in which strong and opposing views prevail, the truth will probably be found somewhere between the extremes. I suggest the following as the rational lines to follow in dealing with ulcer cases.

A. For all acute ulcers, for those which have not caused more than six recurrent attacks of symptoms, for those which give rise to no gross deformity in the radiogram and show no signs of true penetration, for those which have not given rise to more than one serious haemorrhage, and for those in highly nervous subjects, medical treatment should be given a fair and prolonged trial.

B. For all chronic ulcers causing gross deformity and organic stenosis, for all cases where more than six attacks have occurred, where strict dietetic regime is impossible, where there is definite $x$-ray evidence of serious organic deformity, for all the penetrating gastric ulcers, and particularly for those in which the $x$-ray photograph discloses a crater judged to le $1 \mathrm{~cm}$. or more in diameter, surgery is demanded and medical treatment unjustifiable. For all ulcers which have bled freely on three or more occasions surgery must be considered.

The Conservative Strgery of Utcer.

How may we give the unquestionable benefits of surgery to the groups of cases indicated above, and at the same time aroid the unpleasant, and indeed dangerous, complications of regurgitant vomiting and jejunal ulcer, whilst conserving as much normal tissue as we can? For the duodenal ulcer with marked stenosis a posterior gastroenterostomy, placed not too far to the left and with a rcomy aperture in the mesocolon, cannot be improved on; the results are consistently good.

Perhaps the most impressive review of the results of the conservative surgery of duodenal ulcer is that given by Balfour, who followed up the cases of onc hundred medical men operated on in the Mayo Clinic for duodenal ulcer. The average time since operation was eight and a half years. A gastro-enterostomy had been done in 62 per cent. of the cases, excision in 6 per cent. and gastro-duodenostomy in 2 per cent. In 90 per cent. the result of the operation was complete relief from symptoms, and in the great majority the reports were almost extravagant in their praise of the effects of the operation.

For the persistently recurring duodenal ulcer without much stenosis or gastric dilatation-the type which we feel should be cured by medical measures, as it often is for a short time-in the man who cannot take any liberties without suffering and will not lead " the life of a weaned child," for this type a gastro-enterostomy always carries with it a potential jejunal ulcer, and a gastro-duodenostomy is undoubtedly the operation of choice.

\section{Gistro-duodenostomy for Duodenal Ulcer}

In the last few years I have gradually substituted this operation for posterior gastro-enterostomy in all cases without very definite stenosis. At first I practised it mainly in female patients with a lax, readily mobilized duodenum, but latterly I have found that it can be done almost as easily and with equal advantage in the male. The rationale of the operation is that it excludes from the food stream the ulcer-bearing area of the duodenum; that the new opening is in the pyloric end of the stomach, the peristalsis of which is not interfered with; that the acid gastric contents are discharged into the second part of the dundenum directly opposite the point of entry of the bile and pancreatic juice; that the rest of the small intestines need not be disturbed, and incidental complications from achlesions, etc., are thus minimized; that the ulcer or ulcers are left untouched, a direct attack upon the average duodenal ulcer being, in my opinion, quite unnecessary.

The essentials for the operation are complete and effective mobilization of the second and the commencement of the third parts of the duodenum. To obtain this the hepatic flexure of the colon must ke freed, the peritoneum on the outer side of the second part of the duodenum incised, the fascial bands, which hold down the second and especially the third parts of the duodenum, divided, and the duodenum brought to the surface. Morison's pouch is then packed off, and the anastomosis carried out with two layers of No. 00 catgut sutures. It is better to do the operation without clamps, as the less the duodenum is traumatized the better. In this operation the pylorus and first part of the duodenum are folded the one on the other, making an effective barrier to the passage of food through the pylorus. In the few cases where satisfactory mobilization of the duodenum has been impossible a Finney's opcration has been performed with almost equally good results.

It may be urged against this operation that the postoperative course is frequently not so smooth as that of a posterior gastro-enterostomy. Against this, however, may be claimed the infinitely smaller risk of a peptic stomal ulcer. I have not encountered any, and I have only heard of two. The operation disturbs the normal physiology less than does a gastro-jejunostomy, and the possibilities of late complications are infinitely less. When the stomach is grossly dilated or markedly ptosed it does not give tho effective drainage obtained by a gastro-jejunostomy, and in one case where drainage was unsatisfactory $I$ had subsequently to perform the latter operation, which gave complete relief.

\section{Surgical Treatment of Gastric Ulchr.}

When an ulcer has actually penetrated the muscular coats of the stomach and is adherent to the pancreas, and especially when it is associated with one or more duodenal ulcers-which it is in over 50 per cent. of cases in my series-the chances of sound healing and lasting cure ly medical measures are exceedingly small. Without in any way wishing to labour the question of malignant degeneration of simple gastric ulcer, and while considering that it is a relatively rare complication, I believe that it occurs sufficiently of ten to make long-continued medical treatment unjustifiable. It is well-nigh impossible to obtain reliable figures on the question. Two cases, however, where the course from simple ulceration to malignancy was observed, impressed we with the danger. In one case a man over 50 years of age had been under medical treatment for over eight years for what was diagnosed as duodenal ulcer. He came to operation on account of jaundice associated with a palpable swelling below the right costal margintaken to be the gall-bladder. I found a healed duodenal ulcer and a large gastric ulcer, not palpably malignant, but associated with a large secondary malignant nodule in the liver. The edges of the ulcer were found to be infiltrated by malignant disease.

The second case was in a woman of 28 who, two years previously, had had a gastro-enterostomy performed for what appeared to be a simple peptic ulcer of the lesser curvature. When I saw her, two years after her operation, she had a palpable mass in the epigastrium. This proved to be a malignant mass on the lesser curvature spreading from the edges of a penetrating ulcer.

I believe that large gastric ulcers should be excised. Whether the operation be a partial gastrectomy or a free local excision with a gastro-enterostomy, a gastro-duodenostomy or a Finney's operation is, I think, a minor technical matter. Personally I prefer to do a local excision with a gastro-duodenostomy. This ensures free drainage from the stomach, and excludes the duodenal ulcer which is so often present as well, whilst conserving normal tissue and maintaining an attitude of respect for what, after all, cannot but be a useful organ.

The Cautcry Method.-When an ulcer is found high on the lesser curvature and of no great size, the destruction of the base and floor of the ulcer by means of the thermo-cautery along with a short-circuiting operation, as introduced by Balfour, is a method which in my hands has given very good results. For larger ulcersthat is, for those in which surgical treatment is most clearly indicated-excision is, I believe, undoubtedly preferable.

Cholecysto-gastrostomy.-The bilification of the stomach by anastomosing the gall-bladder to that organ, advocated and practised in this country by Braithwaite, is based on 
the conception that the neutralization of the acid gastric juice by the regurgitant fluid through a gastro-enterostomy is due mainly, if not entirely, to the bile contained in that fluid. Whilst good results have been reported in cases of gastric ulcer so highly situated in the lesser curvature as to preclude excision, and in a number of cases of doubtful pathology with hyperacidity as their dominant symptom, the basis of the method is insecure, as bile in itself is not alkaline, and after a gastro-intestinal anastomosis it is the duodenal and pancreatic secretions rather than the bile which give the regurgitant fluid its neutralizing properties. I have carried out this operation in only two ulcer cases, one of very high lesser curvature ulcer and one of gastro-jejunal ulcer. In both cases some immediate improvement resulted, but the symptoms of acidity and pain returned.

Ulcer as One of Many Septic Foci.

No treatment of ulcer can bear the promise of lasting cure if it does not take cognizance of the other foci of infection which accompany it. The prevalence of dental infection is to my mind the most important single factor in determining the frequency of peptic ulcer. It is indeed surprising to find in the vast majority of the patients sent for surgical operation after ineffective medical treatment that gross dental infection has been left untreated. In many of these cases we recover from the pyorrhoeal pockets the same streptococcus as we find in the ulcer. Efficient dental treatment must, therefore, be a first consideration, no matter what other lines of therapy we may follow.
It is within the abdomen, however, that we so frequently find other infective foci which must be dealt with at the same time as the ulcer. The combination of an ulcer in the stomach or duodenum or both with infection in both gall-bladder and appendix is quite common. All three lesions must be dealt with if complete and lasting relief is to be obtained. The following brief note of a case will illustrate the significance of a thorough eradication of foci.

Mr. A. B., aged 57, had thirteen years previously had a gastroenterostomy performed for duodenal ulcer. He obtaincd some temporary relief, but his old pain soon returned; he suffered from nausea, flatulence, and constant abdominal discomfort, and led the life of a ehronic dyspeptic. On examination we found him emaciated, with a dilated stomach and tenderness over three points in the abdomen-just to the left of the umbilicus, just below the right costal margin, and in the right iliac fossa. $X$-ray examination costal margin, and in the right iliac fossa. $\boldsymbol{X}$-ray examination
showed his stomach to be dilated, the stoma stenosed and irregular, showed his stomach to be dilated, the stoma stenosed and irregular,
evidently from a jejunal ulcer. At operation a jejunal ulcer was found and exeised and his stoma enlarged. His gall-bladder was thickened and inflamed, as was his appendix; both were removed. Regional lymph glands were taken from all three sites and from all the streptococcus viridans was grown in pure culture. On injection into rabbits both cholecystitis and duodenal ulcer were produced. Since the second operation the patient has enjoyed perfect heaith and lost all his dyspeptic symptoms.

It may be that we shall eventually succeed by suitable inoculation in immunizing patients against these multiple streptococcal abdominal lesions, or, still better, by timely dental treatment and better dietary and hygienic treatment, in preventing them. Till then, however, surgery must continue to play a part in dealing with the full-blown lesions, and surgery, whilst conserving healthy tissue, must deal thoroughly and radically with the different foci.

\section{An Address on \\ RADIUM AND ITS SURGICAL APPLICATIONS.}

Given before the Bristol Medico-Chircrgical Society BY

H. S. SOUTTAR, M.D., M.CH., F.R.C.S., SURGEON, LONDON HOSPITAL.

R.spium was discovered by Madame Curie in 1898, and its discovery marks an epoch in the history of science. It destroyed at one blow the conception of the atom as an indestructible unit, upon which the whole of chemistry and the greater part of physics had been built, and it opened up a field of investigation which has provided work for physicists for the last thirty years, and has led to discoveries which have revolutionized all our ideas of the physical universe.

Soon after the element had been isolated Becquerel, by inadvertently carrying radium in his pocket, discovered its destructive action on the human tissues, but although attempts were made to turn this to surgical advantage, it was only in isolated cases and in special regions that effective results were obtained. Whilst fully recognizing the value of the work done with radium in diseases of the skin and in carcinoma of the cervix, one must admit that only lately have the surgical possibilities of radium been appreciated, or any attempt been made to exploit its powers. Indeed, those who have had the opportunity of studying the subject must feel that we are only at the beginning of what may prove to be a new epoch in the treatment of malignant disease. For the first time in the history of medical science we hold in our hands a power which beyond any question can cure cancer in many of its forms, and which can destroy a malignant growth without its surgical removal, and it is our business to explore its possibilities to their utmost limits.

This is a subject which none of us can afford to neglect, and it is essential that every surgeon should realize what can be done with radium and understand the methods by which it is applied. To use it effectively he must know something of the physical properties of the new weapon which has been placed in his hands, and I therefore make no apology in describing briefly those physical properties which are fundamental in the action of radium when it is used by the surgeon.

The revolutionary conception involved in the discovery of radium was the idea that an element could decay, and in the process of decay be transformed into an entirely different substance. It has been established, however, beyond any possible question that certain elements possess this power, and they can indeed be arranged in a number of definite series in which each element is the product of the last. The most important of these is the uranium series in which radium occurs, and you will see the parentage and descendants of radium set out in the family tree shown in Table I. Not only do we know its family

Table I.-The Uranium Series.

\begin{tabular}{|c|c|c|c|c|c|}
\hline \multicolumn{3}{|c|}{ Element. } & $\begin{array}{l}\text { Atomic } \\
\text { Weight. }\end{array}$ & Radiation. & Half-value Period. \\
\hline Uranium 1 & $\ldots$ & $\ldots$ & 238 & $a \beta \beta$ & $5,000,000, c 00$ years. \\
\hline Uranium 2 & ... & ... & 234 & $a$ & $2,000,000$ years. \\
\hline Ionium ... & $\ldots$ & ... & 230 & $a$ & 140,000 years. \\
\hline Radium ... & ... & $\ldots$ & 226 & $a(\beta)$ & . 1,750 years. \\
\hline Radon ... & ... & $\ldots$ & 222 & $\alpha$ & 3.85 day's. \\
\hline Radium A & $\ldots$ & ... & 218 & $a$ & 3 minutes. \\
\hline Radium B & ... & $\ldots$ & 214 & $\boldsymbol{\beta}(\gamma)$ & 26.7 minutes. \\
\hline Radium C & $\ldots$ & $\cdots$ & 214 & $\alpha \beta \gamma$ & 19.5 minutes. \\
\hline Radium D & $\ldots$ & $\ldots$ & 210 & $\boldsymbol{\beta}$ & 17 years. \\
\hline Radium E & $\cdots$ & $\ldots$ & 210 & $\boldsymbol{\beta}$ & 5 days. \\
\hline Polonium & $\ldots$ & $\ldots$ & 210 & a & 136 days. \\
\hline Lead & $\cdots$ & $\ldots$ & 205 & - & - \\
\hline
\end{tabular}

history, but we know the expectation of life of each of its members, as represented by its half-value period, the time in which one-half of the element has disappeared, and you will see that this varies considerably, since while uranium 1 attains the patriarchal age of five thousand million years, radium itself is half gone in 1,750 years, and radium $A$ in three minutes. It is indeed to this rapid decay of radon and the other elements in the centre of the table that we owe the surgical possibilities of radium. There is something which stirs me profoundly in this dry table. The 